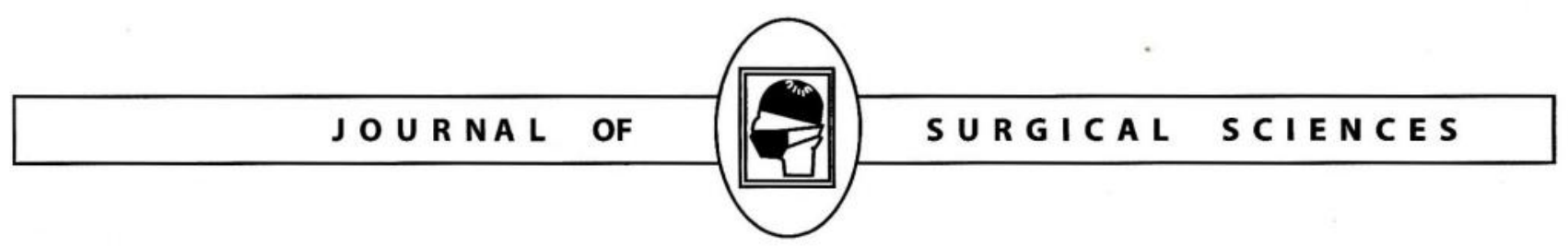

\title{
BILE DUCT INJURY DURING LAPAROSCOPIC CHOLECYSTECTOMY: RISK FACTORS, MECHANISM AND PREVENTION.
}

\author{
Md. Ali Akbar \\ Retired professor of surgery. \\ Comilla Medical College. Comilla.
}

Gall stone disease is one of the most common condition encountered in general surgical practice in adult population. ${ }^{1}$ Laparoscopic cholecystectomy (LC) has replaced open surgery in the treatment of cholecystolithiasis due to its less postoperative pain, better cosmesis, shorter hospital stays and disability. It is now considered the first option and has become one of most common operation performed world wide. $^{2}$

In our country laparoscopic cholecystectony was first performed on December 1991, at BIRDEM hospital by a group of surgeon led by Dr. Hashimoto of Japan. Since then it has gained wide acceptance in our country and is being practiced in almost all medical colleges, district hospitals and most of the private clinics even at upozilla level. The increasing popularity of $\mathrm{LC}$ and patients demand have made the younger generation of surgeons who are even less familiar with open cholecystectomy to come forward to perform this procedure.

Bile duct injury is a severe and potentially life threatening complication of LC. Although there is no definite statistical data of bile duct injury available in our country, the incidence of $\mathrm{BDI}$ is increasing evidenced by increased number of cases in referral hospitals.

The causes of laparoscopic BDI are multifactorial, including:

- Factors inherent to the laparoscopic approachincludes the limitation of two-dimensional vision, the loss of manual palpation of the porta hepatis, the use of tangential and inferior approach to the common bile duct giving suboptimal visualization of the common hepatic duct, the increased difficulty in the control of bleeding, the surgeon's dependence on the material and on the camera holding assistant, the blind manipulation of the instruments,
- Local anatomical risk factors for BDI- acute cholecystitis, small fibrosed gallbladder, scarring in the Calot's triangle, bleeding obscuring the operative field, a huge impacted gallstone within the Hartman pouch, a short or absence of cystic duct and some anatomical anomalies.

- Inadequate experience and training of surgeonThe laparoscopic "learning curve" of the surgeon is a key-factor contributing to the high rates of bile duct injury.

- Poor visualization due to use of poor quality instrument and poor assistance by camera holder

- Thermal injury related to extensive use of monopolar cautery near the portal hilum, during dissection of the Calot's triangle or during attempt to stop haemorrhage.

- The lack of adequate conversion to a more safe open approach in difficult cases.

A clear delineation of junction of the cystic duct with gall bladder is identified by cystic node of Lund with the demonstration of a space between the gall bladder and liver clear of any other structures other than cystic artery called the safety window or critical view has already been recommend as an essential step to prevent injury during laparoscopic cholecystectomy. ${ }^{3,4}$

Safe laparoscopic cholecystectomy needs

1) a good selection of cases,

2) achieve a clear vision,

3) Proper and clear identification of anatomical landmarks and

4) to follow a safe technique of dissection to remove gall bladder. Here are some suggestions for new generation laparoscopic surgeon to perform safe laparoscopic cholecystectomy:- 
- Difficult cases should be avoided early in one's experience. Patients who are male, elderly, when there had been repeated attacks of pain and contracted thick walled gallbladder on USG are considered to be difficult.

- To achieve a clear vision good quality instruments are to be used. They can make group (team) to afford the high cost. Take another surgeon as 1st assistant (camera holder) who can help to get clear vision.

- Because cystic duct and artery are the structures to be divided, it is these structures and these structures only that must be conclusively identified in every laparoscopic cholecystectomy. To achieve conclusive identification, Calot's Triangle must be dissected free of fat, fibers, and areolar tissue.

- Dissection to be started near the neck of the gall bladder (cystic lymph node is an important land mark) and the lower end of the gallbladder dissected off the liver bed then proceed from the lateral to the medial direction, keeping close to the gall bladder.

- Freeing the posterolateral attachments of the gall bladder to the liver creates a good window and the junction of the neck of the gall bladder and the $C D$ is defined all round.

- The Rouviere's sulcus is a fissure on the inferior surface of the liver between the right lobe and caudate process and is clearly seen during posterior dissection of Calot's triangle. Dissection is to be done above and infront of this sulcus to avoid bile duct injury.

- A $30^{\circ}$ telescope is preferable as it can be turned to achieve an en face view of the Calot's triangle. Withdrawing the telescope intermittently gives the surgeon an overall perspective and spatial orientation.

- Clips are to be placed close to the gall bladder after proper visualization of both their limbs. A short or wide CD should preferably be tied.

- Avoid excess traction on infundibulum of GB while applying clip to avoid tenting injury.

- Clips should not be applied blindly and hurriedly to stop bleeding in Calot's triangle.
- Excessive and unnecessary dissection or use of electrocautery near the CBD to be avoided. Cautery to be used at very low power setting in Calot's triangle.

- Electrocautery on tissues close to metal clips concentrates thermal energy and desiccates the tissue making the clips less secure predisposing to bleeding and biliary fistula and hence should always be avoided.

- Any bleeding should be controlled only after accurate identification of its source and the neighboring structures.

- Always better to seek the opinion of senior colleagues of the same institution.

- If one feels "lost"; if doubt persists convert to open cholecystectomy-it only shows good judgment.

In case of difficult cholecystectomy resulting from extensive adhesions, acute cholecystitis, long standing chronic cholecystitis, small contracted gall bladder, and fibrosed and obliterated Calot's triangle, early conversion from laparoscopic to open operation is advised. The negative effects of conversion or even aborting the procedure are minor compared with the negative effect of BDI. Conversion to open procedure, proceeding with partial cholecystectomy, or even aborting the operation and placing cystostomy tube are all viable and acceptable options to decrease the risk of $\mathrm{BDI}$.

\section{References}

1. Abbasi SA, Azami R, Haleem A, Tariq GR, lqbal A, Almas D, et al. An audit of laparoscopic cholecystectomies performed at PNS Shifa. Pak Armed Forces Med J 2003;53:51-8.

2. Targarona EM, Balague C, Knook MM. Laparoscopic surgery and surgical infection. $\mathrm{Br} \mathrm{J}$ Surg 2000;87:536-44.

3. Hermann RE. A plea for a safe technique of cholecystectomy. Surgery 1976;79: 609-611.

4. Yushi T, Henchi I, Ken K, Yukio Y, Masahiko O. Introduction of a 'safety zone' for the safety of laparoscopic cholecystectomy. Am J Surg1993; 88:1258-1261. 